\title{
Creating a Virtual Biomechanics Camp that Emphasizes Socially Derived Learning Communities
}

\author{
G. R. Burks, ${ }^{1}$ L. C. Hebert, ${ }^{2}$ and J. R. Amos (i) ${ }^{1}$ \\ ${ }^{1}$ Department of Bioengineering, University of Illinois at Urbana-Champaign, Urbana, IL, USA; and ${ }^{2}$ Grainger College of \\ Engineering, University of Illinois at Urbana-Champaign, Urbana, IL, USA \\ (Received 24 June 2020; accepted 21 July 2020; published online 17 August 2020)
}

\section{CHALLENGE STATEMENT}

In March 2020, many K-12 students were told to go home and $\log$ onto their computers with no sign of returning to school in the spring. Students received a variety of different experiences ranging from packets of worksheets to several live Zoom calls during the week with their teacher to continue learning the curriculum. Through this experience, we learned that there is no smaller classroom than a family's dining room and there is no better way to personalize instruction than through a parent or other family member.

Similar to transitioning teaching and learning to a virtual environment, the logistical issues that arise through the process of converting traditional face to face summer camps into fully virtual summer camp experiences are many. Some of the important issues to consider are relevant to both in-person and virtual camp contexts, such as:

1. Providing appropriate value to justify the expense,

2. Facilitating an authentic bioengineering experience,

3. Ensuring camper interest and excitement such that they start conversations at home about the content and experience - extending the STEM pipeline and supporting student agency, and

4. Providing enough meaningful opportunities for peer-to-peer interaction to support the formation of effective and lasting learning communities.

In addition, the planning team asked the following questions relating to the format of the camp:

Address correspondence to J. R. Amos, Department of Bioengineering, University of Illinois at Urbana-Champaign, Urbana, IL, USA. Electronic mail: jamos@illinois.edu
1. How can these practices be scaled to accommodate large amounts of students?

2. How should camp scheduling be structured to accommodate the unique home environments of students participating?

3. How can students obtain help and support when they run into issues?

4. How to provide the social spaces and facilitation that support the formation of learning communities and foster environments for STEM inclusion in spite of virtual isolation?

The planning team agreed that it was this final question of a socially-derived learning community that was key to offering an impactful, memorable, and enriching camp experience while also being one of the most challenging to address. Here a socially derived learning community is an interpretation of social learning theory whereby vicarious and shared learning experiences from a model play an important role in reinforcing learned concepts and operates under the assertion that a large part of the student learning experience in a traditional school setting comes from their observation of and interactions with peers. ${ }^{1}$ In a traditional virtual learning environment where the student is the only person engaged with the virtual classroom, observational learning that would usually come from interactions with students around them can be lost, especially when the participation of others is not explicitly called into action by the given learning activity. When moving from an in-person camp experience to a fully virtual one, one of the more challenging aspects to transition is the learning and engagement with content that comes from studentstudent interaction and social mimicking of emotional cues that further reinforces information retention through the creation of memorable moments. To begin 
to approach the reconstruction of these socially driven aspects of learning, we rely on our pedagogical design to create a space whereby the formation of a socially derived learning community is made possible. So, we focused our attention on fostering an inclusive, interactive STEM environment in an otherwise isolated virtual learning space and on providing value for students from a virtual pedagogical design point of view.

Many of the existing science, technology, engineering, and mathematics (STEM) engagement opportunities operate on a "deficit model" form of science outreach. ${ }^{8}$ Camps are often unidirectional and share new knowledge with the public, under the assumption that the public would become more engaged with science if they just knew more of it. ${ }^{4}$ Our camp aims to consistently engage campers on the relationship to what they are doing in their lab exercises to the real world. To this end, the Bioengineering camp has always aimed to show the campers the broad, social implications of engineering. ${ }^{4}$ Underlying this desire to connect science, engineering and society was our hope that young prospective engineers could be recruited to be a generation that prioritizes an inclusive environment. In addition, we see this approach as adaptable to the K-12 classroom for more engaging and inclusive STEM education.

\section{NOVEL INITIATIVE}

Prior to COVID-19, the week-long bioengineering camps provided two of fifteen residential camps offered by the University of Illinois' Grainger College of Engineering for high school students from across the U.S. These engineering camps either provided a deep dive into a particular field of engineering for a full week (50 campers attending bioengineering) or offered campers an opportunity to briefly sample each major through half-day experiences with each field (providing a brief introduction to bioengineering for 100 campers). With the pivot to a virtual camp to provide a combination of the light-touch introductions to all of the engineering majors and an opportunity to go a little deeper into a few topics of choice, the bioengineering department would now touch 475 virtual campers $(44 \%$ identifying as female or non-binary, $14 \%$ black or mixed race, $12 \%$ Hispanic/Latinx), with 135 campers attending bioengineering's "deeper-dive" mini-courses.

For the introduction to bioengineering activity, we introduced human biomechanics and motion capture analysis to students through the merger of free online computation tools (ImageJ), a popular social media theme, family-oriented engineering challenges, and reflection. The novelty in our approach is in the peda- gogical design and how these components work together to foster an authentic engineering learning environment within the context of an individual student's home and to impart a sense of agency in those same students.

During the COVID-19 pandemic, the use of TikTok (C) to record and share videos of popular dances emerged as a way for families to bond and pass the time. ${ }^{5,7,14}$ For this virtual lab, participants are asked to film themselves performing a TikTok@ dance with markers on their head, shoulders, hips, knees, ankles, and hands. This video is then loaded into ImageJ. The TrackMate plugin is used to identify and track the markers to create a $\log$ of 2 dimensional $(x, y)$ movement in the program. After the participant tracks his or her own movement, they can track other family members or friends. Campers are tasked to determine who is the "best" dancer by comparing the smoothness of movement, range of motion, and other characteristics of their dancing ability (Fig. 1 and see supplementary analysis document for more analysis details).

This activity is then used to talk more about biomechanics and how bioengineers need to study and understand biomechanics in order to design orthopedic braces or prosthetics for patients with differing physical needs.

In the supplemental information, you will see the step-by-step guide that was provided to the students in written form. They also received a video introduction to the activity providing $25 \mathrm{~min}$ of explanation and screen-captured instructions for successful setup and use of the software. All of these materials were housed in a learning management system for campers to access at their convenience and intended to take a maximum of $1.5 \mathrm{~h}$ to complete. Additionally, the instructor offered scheduled office hours for a synchronous opportunity for campers to ask questions or to get help with the issues they encountered, using the video conferencing software.

\section{REFLECTION}

Using popular culture themes to teach scientific concepts is a well-known pedagogic strategy. Previous outreach programs have asserted that science outreach impact is lessened unless it can capture and hold the attention of the audience. ${ }^{9}$ To this end, the primary goal of the summer program was to use pop-culture themes to broaden the appeal of scientific topics. With the uprise of TikTok ${ }^{\circledR}$ videos during the COVID-19 pandemic, it seemed a likely way to engage youth and their families into scientific and engineering concepts; however, it is important to note that it is not required that students have a formal account on TikTok ${ }^{\circledR}$ or that students use $\operatorname{TikTok}^{\circledR}$ at all to complete this 

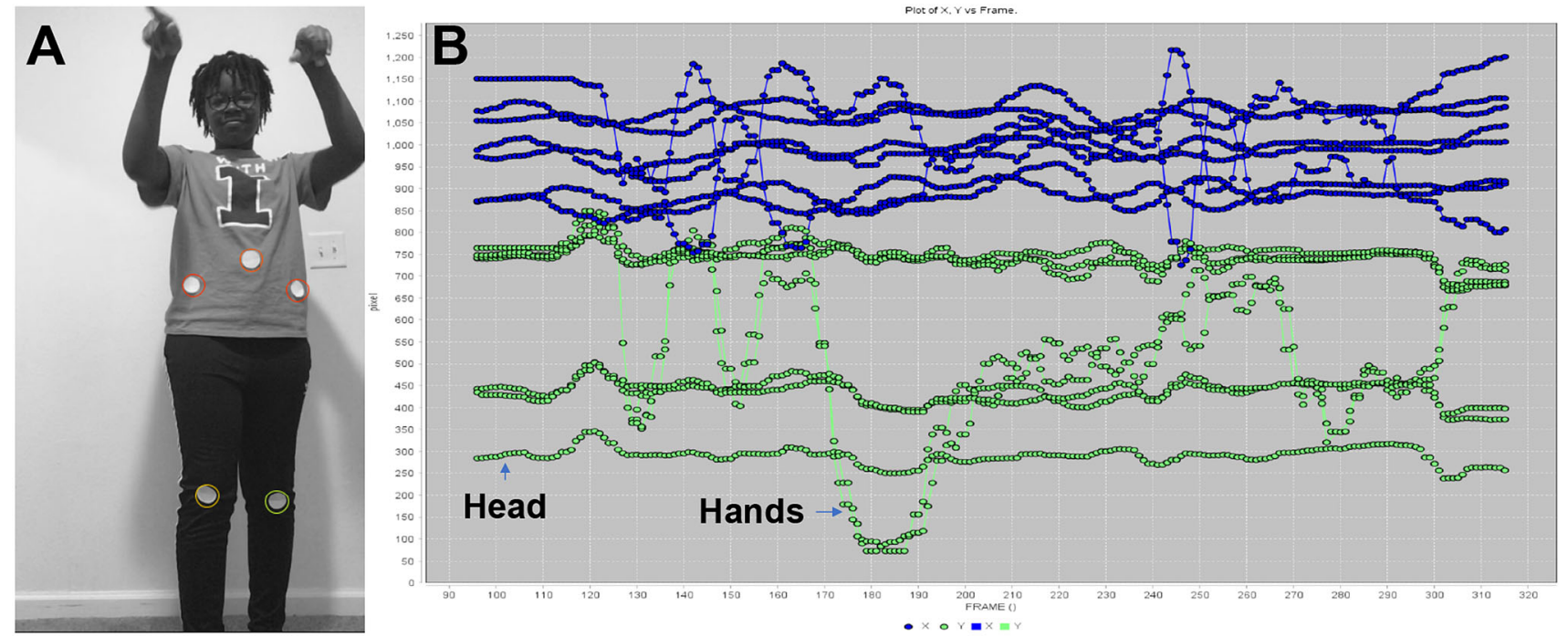

FIGURE 1. (a) A model student recording a dance and tracking their motion using ImageJ TrackMate. (b) Motion tracking data analysis from TrackMate that shows $x$ - and $y$-displacement curves for dots tracked. In (b), the green curves represent $y$ displacement, blue curves are $x$-displacement, the $x$-axis the frame count, and the $y$-axis is in pixels (could be converted to inches by including a calibration mark in the recorded video).

activity. Given the unpredictable variance that may come through adolescent exposure to social media platforms, it is important to impart that students have options to engage with any dance culture to provide content for their personal conduction of motion analysis.

During the execution of this activity, two of the common challenges for students were: (1) having access to computer hardware capable of handling the demands for installing and running the required software, and (2) establishing sufficient dance models from which their dancing abilities could be compared analytically. With respect to the technological demands, Image $\mathbf{J}$ is a widely used program but involves frequent updates and patches. This presented issues with computers not being able to meet those updated demands and some even having issues with administrative download restrictions placed on their devices by the schools before issuing the computers to students for remote learning. As it relates to having a model dance to base student dancing abilities off of, the authors had to digitally place tracking dots on a prerecorded video to standardize the model dancing profiles. This is a capability that most may not have; therefore, to run this activity effectively it will be important that explanation and standard dance models be made available to the students so that they can conduct their analysis in the least biased way possible.

This activity seemed particularly successful at engaging students with their household members and engaging those other household members in the engineering learning process. The students were able to better draw conclusions regarding the differences in people with differing physical builds and biomechanical limitations through working with family members who may have been older, younger, or who possessed different injury histories than themselves. Their experiences engaging with household members added much value to our group reflection time during the follow-up portion of the camp.

Research by Bell, Lewenstein, Souse, and Feder in 2009 showed that informal learning has a range of outcomes that can supersede traditional learning in an academic setting. ${ }^{2}$ A traditional classroom setting is not an environment restricted to one-on-one interaction, and therefore the dynamics and perceptions of multiple relationships should be considered ${ }^{6}$; furthermore, much of the learning in these settings is invisible and taken for granted, and thus, is hard to measure. ${ }^{15}$ Further still, there is a hidden social meaning-making process in informal learning that can be missed if not designed in a culturally supportive way for a community. ${ }^{15}$ The pedagogic approach used here attempts to foster opportunities for the social meaning-making process and to develop learning rapport between participating students and their respective household members by explicitly soliciting the participation of those household members into the engineering activity process. The camp provided additional tools and strategies that campers were encourages to use to form organic study groups, to share and discuss activity processes and outcomes including a camp-specific chat platform and small group assignments that were consistent throughout the duration of the camp. While a handful of students used these spaces for discussing the lab activities, the majority of participants used their 
family members as their learning community for this activity. This design circumvented the typical isolation associated with virtual learning. It can be expected that the student and their respective household member(s) may be coming into the learning space with differing experience levels with the content, in which case it has been shown by Nelson and DeBacker that spending time to develop interpersonal student-student rapport yields a peer climate conducive to achievement, belongingness, and academic efficacy. ${ }^{13}$ In a familyoriented and accessible STEM learning context, establishing activity participants as learning peers and fostering a sense of belongingness amongst those who the camp activity is not primarily designed for are of great importance to further support the learning efficacy of the student camper. In these cases, the camper took on an instructional, facilitation role to bring family members into the shared lab experience.

McClellan developed a model for the process of individual learning in informal settings where the learner must choose to transform existing knowledge, hinting at the influence of community learning and a sociocultural perspective. ${ }^{12}$ Building on this theory, Marsick \& Watkins emphasized that negotiation that occurs in informal settings can lead to disruption of held beliefs and encourages intermixing of beliefs. This then will lead to a new commonly held belief within the group. ${ }^{11}$ This informal learning model from Marsick and Watkins claims that groups will go through a series of steps to reconcile concepts within a social context, building to a group held belief before entering into the activity of the group as a whole. ${ }^{11}$ This is applicable here, in that challenges will inevitably arise for students as they proceed through the bioengineering activity-during which time they will form their own interpretations and beliefs about the provided content — but having an accessible household learning community gives rise to opportunities for group context building, reshaping of those newly formed previously held beliefs, and establishing meaningful rapport through shared struggles with content. It cannot be understated the value in renegotiating the meaning of complex STEM content among a group of co-learners, whether this means family members of peers.

Another theoretical principle that informs this program is Vygotsky's zone of proximal development. ${ }^{3}$ When designing a learning environment, the instructor should attend to all aspects of teaching, particularly its social nature. Vygotsky stressed the importance of experts actively attending to the subtle and not-sosubtle aspects of the learning process. These aspects of learning include cultural funds of knowledge that can be used to motivate students. When discussing the learning process, Vygotsky asserted that higher mental functions emerge in a novice, through social interac- tions with an expert/teacher that are later internalized. ${ }^{3}$ In this unique learning context, the roles of expert and teacher are shared between the student who is interested in potentially becoming an expert in the area and their household members who will have a wide range of social and life experiences that will add to the negotiation of the understanding of the STEM content. Scholars believed that an active student is a motivated student. Therefore, learning how to identify, use, or even create motivation in students is critical for formal and informal educators and should be core to any instructional material. These principles will ensure that we are reaching our target audience, that these families are engaged and learning intended content and skills, and that we have laid the groundwork for their long-term interest.

Finally, we are interested in continuing to evaluate our summer program, but with greater emphasis on understanding it as an educational tool. Due to limitations in time to develop and implement, we did not attempt to assess participant learning, whether through participant self-reflection or more formal assessment. This is critical as research has shown that informal education events play a pivotal role in promoting STEM fields, as well as encouraging and deepening science learning. ${ }^{10}$ Though we developed the summer program in accordance with the National Science Teacher Association (NSTA) recommendations on informal environments and strengthening links between formal and informal science learning, ${ }^{10}$ we intend to more formally evaluate its educational capacity and impact in the future.

We are pleased with what we accomplished in a short time to offer a quality and enjoyable virtual summer camp experience. These simple strategies can be easily employed by other BME educators, and we hope others will feel encouraged to adopt our techniques and materials to launch a similar virtual summer camp at their own institution.

\section{ELECTRONIC SUPPLEMENTARY MATERIAL}

The online version of this article (doi:https://doi.or $\mathrm{g} / 10.1007 / \mathrm{s} 43683-020-00007-y)$ contains supplementary material, which is available to authorized users.

\section{AUTHOR CONTRIBUTIONS}

GB is a postdoctoral researcher who took the lead in writing the article as well as directing the camp activities. LH is the camp manager and was involved in editing of the manuscript. JA supervises Gabriel Burks and also contributed substantial time to writing and editing the manuscript. 


\section{FUNDING}

None.

\section{CONFLICT OF INTEREST}

None.

\section{ETHICAL APPROVAL}

This work was deemed non-human subject research by the University Of Illinois at Urbana-Champaign Office for the Protection of Research Subjects (OPRS) has reviewed and determined that the research study, as described and reported to OPRS, does not meet the criteria for Human Subjects Research. IRB approval is not required. In addition, parental and participant consent was obtained for the photo used in the paper.

\section{REFERENCES}

${ }^{1}$ Bandura A, Walters RH. Social learning theory. Englewood Cliffs: Prentice-Hall; 1977.

${ }^{2}$ Bell P, et al. Learning science in informal environments: people, places, and pursuits. Washington, DC: National Academies Press; 2009.

${ }^{3}$ Chaiklin S. The zone of proximal development in Vygotsky's analysis of learning and instruction. Vygotsky's Educ Theory Cult Context. 2003;1:39-64.

${ }^{4}$ Council NR. Convergence: facilitating transdisciplinary integration of life sciences, physical sciences, engineering, and beyond. Washington, DC: National Academies Press; 2014.
${ }^{5}$ Ezhevskaya A, Arvelo J. Creating Local Arts Together 2.0: building an online community through the global ethnodoxology network. In: Global Forum on Arts and Christian Faith, 2020.

${ }^{6}$ Frisby BN, Martin MM. Instructor-student and studentstudent rapport in the classroom. Commun Educ. 2010; 59:146-164.

${ }^{7}$ Herrman J. How TikTok is rewriting the world. The New York Times, 10, 2019.

${ }^{8}$ Hodson D. Time for action: science education for an alternative future. Int J Sci Educ. 2003;25:645-70.

${ }^{9}$ Kerby HW, et al. Fusion science theater presents the amazing chemical circus: a new model of outreach that uses theater to engage children in learning. J Chem Educ. 2010;87:1024-30.

${ }^{10}$ Learning Science in Informal Environments. An NSTA position statement on learning science in informal environments. https://www.nsta.org/nstas-official-positions/lea rning-science-informal-environments. Accessed 20 Jun 2020.

${ }^{11}$ Marsick VJ, Watkins KE. Informal and incidental learning. New Direct Adult Contin Educ. 2001;2001:25-34.

${ }^{12} \mathrm{McClellan} \mathrm{J}$. Toward a general model of collective learning: a critique of existing models of specific social systems and a sketch of a model for social systems in general, 1983.

${ }^{13}$ Nelson RM, DeBacker TK. Achievement motivation in adolescents: the role of peer climate and best friends. J Exp Educ. 2008;76:170-89.

${ }^{14}$ Omar B, Dequan W. Watch, share or create: the influence of personality traits and user motivation on TikTok mobile video usage. Int J Interact Mob Technol. 2020, 14:121.

${ }^{15}$ Watkins KE, et al., The evolving Marsick and Watkins (1990) theory of informal and incidental learning. New Direct Adult Contin. Educ. 159;2018:21-36.

Publisher's Note Springer Nature remains neutral with regard to jurisdictional claims in published maps and institutional affiliations. 